\title{
Modeling cortisol rhythms in a population-based study
}

\author{
Nalini Ranjit ${ }^{a, *}$, Elizabeth A. Young ${ }^{b}$, Trivellore E. Raghunathanc, \\ George A. Kaplan ${ }^{a}$
}

\author{
${ }^{a}$ Department of Epidemiology, Center for Social Epidemiology and Population Health, \\ School of Public Health, University of Michigan, 1214 South University, Ann Arbor, MI 48104, USA \\ ${ }^{\mathrm{b}}$ Department of Psychiatry and Mental Health Research Institute, University of Michigan, \\ Ann Arbor, MI, USA \\ 'Department of Biostatistics and Survey Research Center, University of Michigan, Ann Arbor, MI, USA
}

Received 19 October 2004; received in revised form 26 January 2005; accepted 1 February 2005

\section{KEYWORDS \\ Cortisol; \\ Diurnal profile; \\ Regression splines; \\ Mixed models}

\begin{abstract}
Summary The aim of these analyses was to develop and describe easily implementable, yet information rich, approaches for analysis of the temporal profile of cortisol in human populations. A typical waking profile of cortisol in a sample of women aged 15-44 was parameterized by a piecewise linear regression model, implemented as a mixed model to accommodate repeated observations among individuals. The model was extended to distinguish characteristic cortisol profiles of obese women from those of non-obese women. Sharp inflection points for the diurnal profile of cortisol were noted at 30 and $75 \mathrm{~min}$ past awakening. Obese women showed a slight tendency to have a less sharply rising and declining response to awakening than non-obese women.

(c) 2005 Elsevier Ltd. All rights reserved.
\end{abstract}

\section{Introduction}

The hypothalamic pituitary adrenal axis (HPA) with its end product of cortisol, is one of several neuroendocrine systems that display profound circadian changes. In the case of the HPA axis, the rhythm is entrained to the wake-sleep cycle so that

\footnotetext{
${ }^{*}$ Corresponding author. Tel.: +1 734615 9216; fax: +1 734 9980006.

E-mail addresses: nranjit@umich.edu (N. Ranjit), eayoung@umich.edu (E.A. Young), teraghu@umich.edu (T.E. Raghunathan), gkaplan@umich.edu (G.A. Kaplan).
}

the highest levels occur shortly after awakening and the lowest levels are found during the early sleep period. Recent innovations in saliva assays have found that saliva cortisol is a useful tool for examining cortisol measures in real life situations (Kirschbaum and Hellhammer, 1994), with some evidence that the magnitude of the morning rise is associated with the level of life stressors (Pruessner et al., 1999). Consequently, assessment of this morning rise may provide a particularly useful tool for examining the biological effects of social and economic stressors on overall health in survey populations, as well as the impact of a number 
of disease and pre-clinical states on the cortisol rhythm.

Epidemiological, population-based studies are beginning to utilize repeated sampling of salivary cortisol with many advantages over the laboratory based assessments of cortisol. As more and more such studies appear, the challenges of modeling cortisol rhythms have become increasingly apparent (Pruessner et al., 2003). Cortisol shows a marked circadian rhythm, increasing about 50-75\% within the first $30 \mathrm{~min}$ after awakening in both sexes, across a wide span of ages (Hucklebridge et al., 2000). Following the morning awakening rise, cortisol levels drop sharply, and then more gradually over the rest of the day, as secretory activity in the HPA-axis declines. In addition, cortisol secretion is pulsatile, and these pulses are superimposed upon the underlying circadian rhythm (Young et al., 2001) contributing additional variability to infrequent sampling of cortisol as utilized in saliva studies.

A variety of approaches have been used to characterize and evaluate the circadian profile of salivary cortisol. These include measures that utilize information on the slope of the decline from the peak to trough levels (Sephton et al., 2000) or alternatively, present separate assessments of morning and/or evening cortisol levels (King et al., 2000; Steptoe et al., 2000). Other studies evaluate total cortisol concentration over the day, using a measure such as area-under-thecurve (e.g. Reynolds et al., 2003). Several studies focus on the rhythm profile of cortisol, as the morning cortisol response to awakening, as well as the extent of decline to the evening nadir can provide important information on the activity of the HPA axis (e.g. Schmidt-Reinwald et al., 1999). At the other end of the spectrum are approaches that seek to describe the cortisol rhythm in all its complexity, and use non-parametric approaches to fit smoothing splines (Erosheva et al., 2002; Wang and Brown, 1996).

Both the modeling approaches and data requirements of these approaches clearly vary. Harmonic analyses such as cosinor analyses enforce a fixed 12 hour period and assume relatively uniform changes on the ascending and descending limbs, which may not be the best fit to the data. Furthermore, these approaches require $24 \mathrm{~h}$ of sampling, which leaves analyses of data from shorter time periods not well addressed. Many analyses rely on ordinary linear regression modeling to model the profile of cortisol data. Such approaches work best with samples evenly distributed over the course of the day, but they do not capture critical aspects of the circadian rhythm of cortisol, including the early morning rise and subsequent decline. Better fit is obtained by using smoothing splines, but these methods, while useful for exploration, do not yield quantitative parameters to allow for group comparison. Also, smoothing splines methods require intensive sampling over the day, and are not practical for use in population-based studies.

Modeling efforts are further hampered by the poor compliance of subjects in sampling at the instructed times. Studies have demonstrated through electronic monitoring of actual times that compliance is over-estimated by reported compliance (Kudielka et al., 2003). In a recent report by Yehuda et al. (2003), involving six samples in a single day, only $49 \%$ of subjects completed all six samples within $30 \mathrm{~min}$ of the specified time. Novel analytic approaches to accommodate such variation in sampling time would therefore be useful.

In this study, we describe simple regression-based approaches for characterizing the waking profiles of cortisol, under conditions when data show a high degree of temporal variability and the number of measures varies across respondents. To illustrate the value of these approaches, the current study analyses repeated measures of salivary cortisol obtained from a sample of poor women aged 2054. A combination of non-parametric and parametric methods is used to arrive at a suitable specification of a piecewise linear regression model with random effects. The specification of the model combines prior theoretical knowledge about diurnal variation in cortisol with the information from the non-parametric descriptive analyses. An illustration of how the analyses can be extended to subject-specific determinants of cortisol rhythms is also presented. Finally, we discuss the implications of our modeling strategy for further data collection and modeling efforts in this area.

\section{Methods}

The participants in this study are drawn from the Women's Employment Study (WES), a random sample of single mothers with children receiving cash benefits as of February 1997, in an urban county in Michigan in February 1997 (Danziger and Seefeldt, 2002). The sample was between the ages of 18 and 54, and either African-American or White. To date, four waves of data have been collected from this sample. In June 2000, following completion of the third wave, a special health supplement (WES-HS) was administered to a representative sub-sample $(n=298)$ of the WES survey respondents. Health measures included up to four salivary cortisol samples, three of which 
were self-administered by the respondents on a single day. Of the four salivary cortisol measures, the first measure was obtained during a clinic or home visit, where the respondent was provided with three labeled Salivettes ${ }^{\circledR \mathrm{тм}}$ and instructed on obtaining salivary samples at home. Respondents were instructed to collect all three samples on 1 day, with the first sample collected upon awakening, the second after $30 \mathrm{~min}$, and the third at bedtime. They were instructed to record the time of sample collection for each sample and to delay breakfast until after the second sample. The collection protocol was designed to capture, with as few measures as possible, critical aspects of the diurnal variation in cortisol, including the morning awakening increase and sharp decline, as well as the more gradual decline to the evening nadir.

Samples were returned by mail to the data tracking center and frozen at $-20^{\circ} \mathrm{C}$ until assay. Samples were thawed and spun in the laboratory immediately prior to assay. They were assayed using DPC Coat-a-Count tubes using $200 \mathrm{ml}$ of saliva per tube following manufacturer's directions. Inter-assay variability was $10 \%$.

\subsection{Analyses}

Analyses presented in this paper are restricted to the 188 respondents for whom at least one valid cortisol measure was obtained, where a valid measure is defined as one that includes both a value for cortisol as well as the time of collection. The correlations between cortisol measures within individuals over time were investigated by estimating Pearson's correlation coefficients between successive cortisol measures. Intra-cluster correlation coefficients were based on an unconditional means model of cortisol level within each woman, implemented using a hierarchical linear model. Non-parametric methods were used to generate a mean profile of diurnal variation in cortisol, and to suggest the functional form and parameters for a fully parametric model. Piecewise linear regressions were tested to help locate knot positions, supplemented by the information available from visual inspection of the individual and mean cortisol trajectories (not shown). To account for the correlation of observations within women, a regression spline model with fixed knots, embedded within a mixed model was used to generate subject specific cortisol profiles. Finally, the model was extended to identify determinants of inter-individual heterogeneity in terms of key parameters of the individual change trajectory (20). All analyses were conducted using SAS (v 8.02).

\section{Results}

At least one valid cortisol measure (defined as a measure that includes both cortisol value and time of collection) was obtained from 188 women, i.e. $63 \%$ of the sample. Non-respondents were similar to respondents on a variety of measures, including age and race composition, and physical health barriers. Among the respondents, compliance with regard to the number of measures obtained was high, so that $70 \%$ of the women provided four measures, and only $7 \%$ of the sample provided no more than one measure. The number of obtained cortisol measures did not appear to vary systematically by a number of respondent characteristics that we examined, including demographic measures, stress measures, and physical health measures. There is little reason to believe that the respondent sample was a selected sub-sample, or that cortisol measures among respondents were not missing at random (MAR). Confirming that the data are missing at random is an important pre-requisite to obtaining unbiased estimates from mixed models of the type that are estimated here.

Nearly $77 \%(n=144)$ of the 188 respondents collected the three home samples; 132 , or $71 \%$ of these also obtained an additional clinical sample. An additional 17 women, or $9 \%$ of the sample, had two measures available for analysis. At the other extreme, 13 of the women, or about $7 \%$ of the sample, had only one usable measure including the clinic measure. In all 651 usable measures of cortisol, nested within these 188 women, were available for analysis. Valid cortisol measures were obtained for 160 clinical samples, 164 awakening samples, 167 second-morning samples, and 160 bedtime samples. While the clinical sample measures were not obtained on the same day as the remaining samples, results from all models presented below were robust to the inclusion of these clinical measures. They were thus retained in all final analyses.

While respondents were able to collect the salivary samples, compliance with the time requirements of sample collection was highly variable. Thus, while respondents were required to collect the first sample immediately upon awakening, the actual mean time of sample collection was about $14 \mathrm{~min}$ after awakening. The second sample, which was required to be collected 30 min after awakening, was collected, on average, about one-and-ahalf hours after awakening. However, the median time gap between the first and second collection is 32 min, suggesting reasonable compliance with this aspect of the protocol. The median gap between 


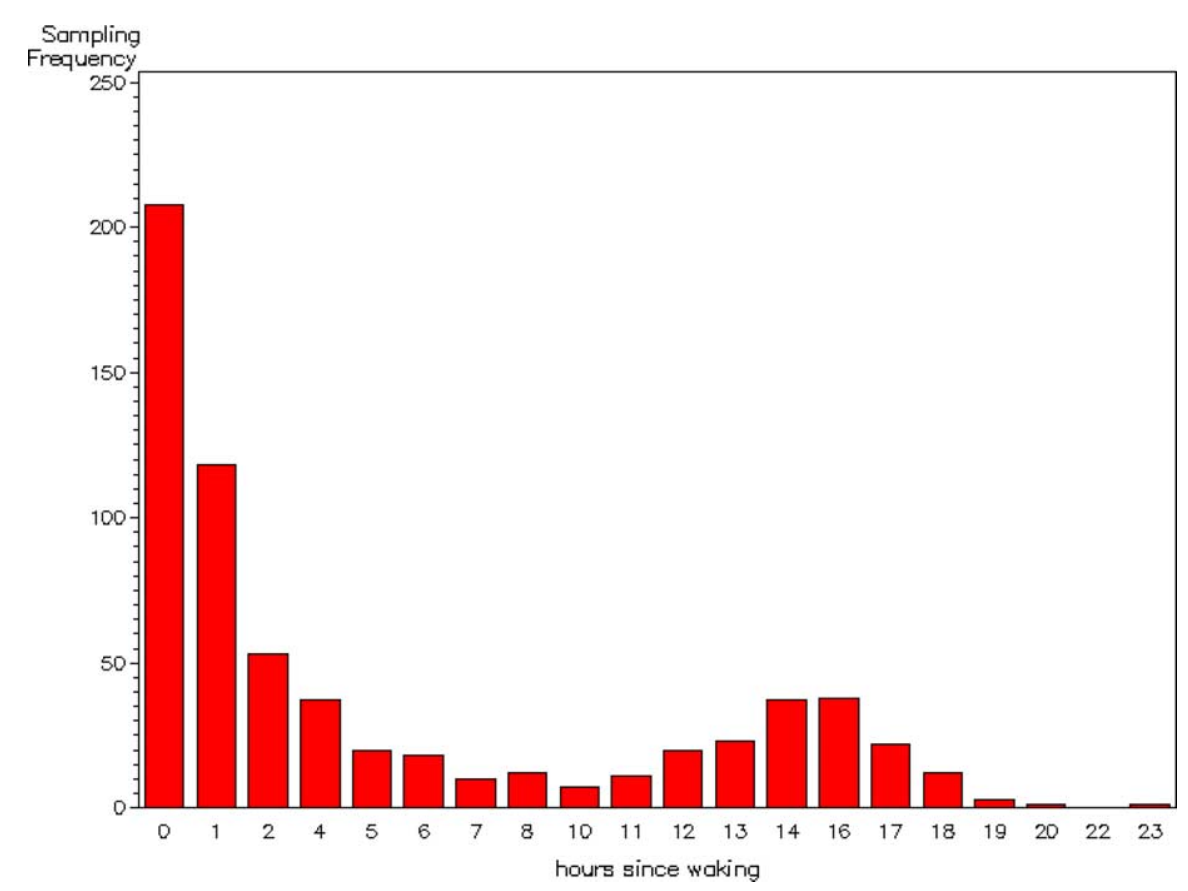

Figure 1 Sampling frequency of cortisol measures.

sample collection times for the second sample and the bedtime sample was about $13 \mathrm{~h}$, with a range from 6 to $19 \mathrm{~h}$. There is consequently a high degree of unplanned variability in sample collection times. The resulting sampling frequency of cortisol values over the day is described in Fig. 1. While sample points are clustered in the first $1.5 \mathrm{~h}$ after awakening, and in the period between 14 and $16 \mathrm{~h}$, as per protocol, it is apparent that a fair proportion of samples were obtained at other times of the day. As it turns out, such temporal variability can be exploited in modeling diurnal rhythms of salivary cortisol.

\subsection{Identifying the mean profile for the population}

A non-parametric loess-based smoother was applied to the obtained cortisol values as a function of time of collection, measured in minutes past awakening. The profile shows a steep rise and an equally rapid decline in the first hour or so after awakening, and then a slower decline to a value that then appears to remain more or less constant for the rest of the measurement period (see Fig. 2 for an example of this pattern). It is evident that a diurnal profile of this form cannot be modeled by a linear or low order polynomial function of time. It can however be readily modeled using some form of spline function. We employed piecewise linear regression models, which are essentially zerodegree spline-based parametric models.
Based on visual examination of the plot in Fig. 2 and theoretical considerations, knots (inflection points) were fixed at $30 \mathrm{~min}(0.5 \mathrm{~h})$ past awakening and $75 \mathrm{~min}(1.25 \mathrm{~h})$ past awakening; in addition, the density of data between 14-16 $\mathrm{h}$ allowed us to test if there was a true inflection at $15 \mathrm{~h}$. When knot locations are chosen primarily on the basis of visual examination of the data, as is done here, confidence intervals obtained from subsequent models have to be adjusted to accommodate this fact. However, for the more limited purpose of description, it is appropriate to present unadjusted confidence intervals. To confirm that these knot locations were appropriate, we tested a simple ageadjusted regression model of cortisol values against a piecewise linear function of time with the 'pieces' defined by these three knot locations (not shown).

The regression confirmed that there was a true change in slope at 0.5 and $1.25 \mathrm{~h}$ past awakening, but not at $15 \mathrm{~h}$. The adjusted $R$-squared of this model was 0.25 , with significant values $(p<0.001)$ on the slope parameters of all three segments. The regression model results were consistent with the graphical profile, suggesting that there are indeed significant sharp non-linearities in cortisol values in the early part of the day, and a relatively linear pattern of decline over the latter part of the day. Fit statistics of the model were robust to small alterations (up to 10 min upward or downward) in the locations of these knots; hence, we retained the knot locations at 30 and 75 min past awakening, as these were the most consistent with the graphical 
pattern. This basic model was extended by adding other variables known or suspected to influence cortisol variation. These included pregnancy status, and smoking or eating prior to each measure. None of the added measures had any important effect on mean cortisol level. In the interests of parsimony and power, the final selected model for the mean included only age (centered at the mean age of the sample, 33.5 years), and a non-linear spline-based function of time (anchored to awakening time) with knots at 0.5 and $1.25 \mathrm{~h}$. Our final model for the mean profile, accordingly, was a zero-degree linear spline with two interior knots, as represented by the following general equation

$=f\left(x_{n-1}\right)+\frac{f\left(x_{n}\right)-f\left(x_{n-1}\right)}{x_{n}-x_{n-1}}\left(x-x_{n-1}\right), x_{n-1} \leq x \leq x_{n}$

where the terms $f\left(x_{i}\right)-f\left(x_{i-1}\right) / x_{i}-x_{i-1}$ represent the slopes between $x_{i-1}$ and $x_{i}$, and $f\left(x_{0}\right)$ is the intercept or awakening value. Three such equations are estimated here, one each for the regions between $0<x \leq 0.5,0.5<x \leq 1.25$, and $x>1.25$, respectively.

\subsection{Modeling subject-specific profiles of cortisol}

Cortisol measures within women are highly correlated, with Pearson correlation coefficients ranging from $r=0.35-0.62$. It is clear that the cortisol measures cannot be modeled as if they are independently drawn from a population of cortisol values; instead, our models have to account for dependence within subjects. Simple regression models can provide correct estimates of a population mean profile, but to the extent that there is within subject correlation, the standard errors of such a model are biased downwards. We describe a series of growth curve models based on modeling subject-specific random effects. The fixed effects model (Level-1) modeled the relationship between individual data points and time since awakening, and is the same as the model for the mean profile as described in the system of equations above. The random specification of the model (Level-2) modeled individual values, and allowed random variation around the population intercept, as well as around each of the three-spline parameters. In the absence of any a priori knowledge regarding the nature of the within-subject correlations, a fully 'unstructured' variance-covariance matrix was presumed. Despite the large number of parameters to be estimated, convergence of the estimation algorithm was obtained within three iterations. The results presented in Table 1 are based on REML estimates, a variant of full maximum-likelihood estimators (MLE).

Based on the results from the random-effects model specification, the mean baseline cortisol level (at start of day) for a woman of average age is $0.38 \mu \mathrm{g} / \mathrm{dl}$. The parameter on the first segment or Phase 1 (corresponding to the morning rise), represents the slope (in units of $\mu \mathrm{g} / \mathrm{dl}$ per hour) of cortisol change from the segment $0-0.5 \mathrm{~h}$ after awakening. Cortisol level rises rapidly during this period at the rate of $0.47 \mu \mathrm{g} / \mathrm{dl}$ per hour. The subsequent parameters model the slope increment in subsequent intervals during the day. Thus, $-0.79 \mu \mathrm{g} / \mathrm{dl}$ per hour, the parameter on Phase 2 (the steep decline following the morning rise, starting at $0.5 \mathrm{~h}$ and continuing up to $1.25 \mathrm{~h}$ ) is the slope increment over the Phase 1 value. Thus, the net slope in this segment is $-0.317 \mu \mathrm{g} / \mathrm{dl}$ per hour $(0.469+(-0.786)=-0.317)$; i.e. cortisol level decreases at the rate of $0.32 \mu \mathrm{g} / \mathrm{dl}$ per hour in the $45 \mathrm{~min}$ after the morning peak. Phase 3 , the interval of slow decline from $1.25 \mathrm{~h}$ to end of day, has a parameter value of 0.302 , or a net slope of $-0.02 \mu \mathrm{g} / \mathrm{dl}$ per hour $(-0.317+$ $0.302=-0.015)$. This implies a slight decline, on average, in cortisol levels over most of the day, after the initial fall from the morning peak has been completed. The age parameter implies a decrease in cortisol level by $-0.049 \mu \mathrm{g} / \mathrm{dl}$, for each yearly increment in age beyond the mean

Table 1 Random effects model estimates of population mean cortisol profile.

\begin{tabular}{lrrr}
\hline Variable & $\begin{array}{l}\text { Parameter esti- } \\
\text { mate and SE }\end{array}$ & $\begin{array}{l}\text { Implied slopes and } \\
\text { SE at knot changes }\end{array}$ & $\begin{array}{l}\text { 95\% Cl of implied } \\
\text { parameter }\end{array}$ \\
\hline Intercept (awakening value) & $0.384(0.026)$ & & $(0.333-0.435)$ \\
Age (centered at 33.5 years) & $-0.049(0.014)$ & & $(-0.076$ to -0.021$)$ \\
Phase 1 (slope of rise over 1st 30 min) & $0.469(0.068)$ & $0.469(0.068)$ & $(0.336-0.602)$ \\
Phase 2 (slope of decline from 30 to 75 min) & $-0.786(0.100)$ & $-0.317(0.040)$ & $(-0.395$ to -0.239$)$ \\
Phase 3 (slope of decline from 75 to 14 h) & $0.302(0.041)$ & $-0.015(0.002)$ & $(-0.019$ to -0.011$)$ \\
\hline
\end{tabular}


age of the sample. The observed parameter values confirm the pattern suggested by the graphical examination. The size of the parameters (as opposed to the small size of the standard errors) suggests that the modeled pattern is not merely due to a greater density of data availability during the early part of the day. The random effects portion of the model results (not shown), confirm significant inter-individual heterogeneity in cortisol profiles, with non-zero variances obtained around the intercept and each of the three slope parameters. The residual of 0.071 obtained from a fixed-effects-only model decreases to 0.027 after inclusion of these women-specific parameters in the model. The difference is accounted for by between-subject variance, which is modeled by the random effects. Approximately, twice the variance in the data is between women, rather than within women. From an unconditional means model of cortisol level, we estimated the intraclass correlation coefficient (ratio of betweensubjects variance to total variance) as 0.62 , which is sizeable. To allow comparison with a fixedeffects-only model, the same model was run with MLE estimates. A decline in the AIC value from 134.7 to -63.9 for this comparison confirms that a model with random effects is markedly better than the model with no random effects specified. Fig. 2 plots predicted values from this model superimposed on the original data values. Overall, the fit is excellent.

\subsection{Associations of cortisol profiles with obesity}

In our next set of analyses, we extended these random-effects models to explore differences in cortisol profile by a subject-specific characteristic. Additions to the basic repeated-measures mixed model at this point involved only changing the specification for the fixed-effects part of the model, not the random effects, since our sample size (number of observations per woman) limited the number of additional estimable random effects. In order to estimate the impact of obesity, we added to the above model, an indicator of obesity status (defined as BMI $\geq 30$ ). Over half of the sample is obese, with 106 women (57\%) classified as obese, compared to 78 classified as non-obese. 260 cortisol observations were contributed by the non-obese women, and 381 by obese women. Thus, there is sufficient power to identify exposure effects along the entire profile.

Exploratory loess-based graphical analyses (Fig. 3) suggest that women's cortisol profiles vary by obesity status. The entire diurnal profile of obese women differs from that of non-obese women, with differences in both the intercept and the various slope parameters as a function of obesity status. The locations of the inflection points, however, are similar for both obese and non-obese women. To examine if these differences by obesity were

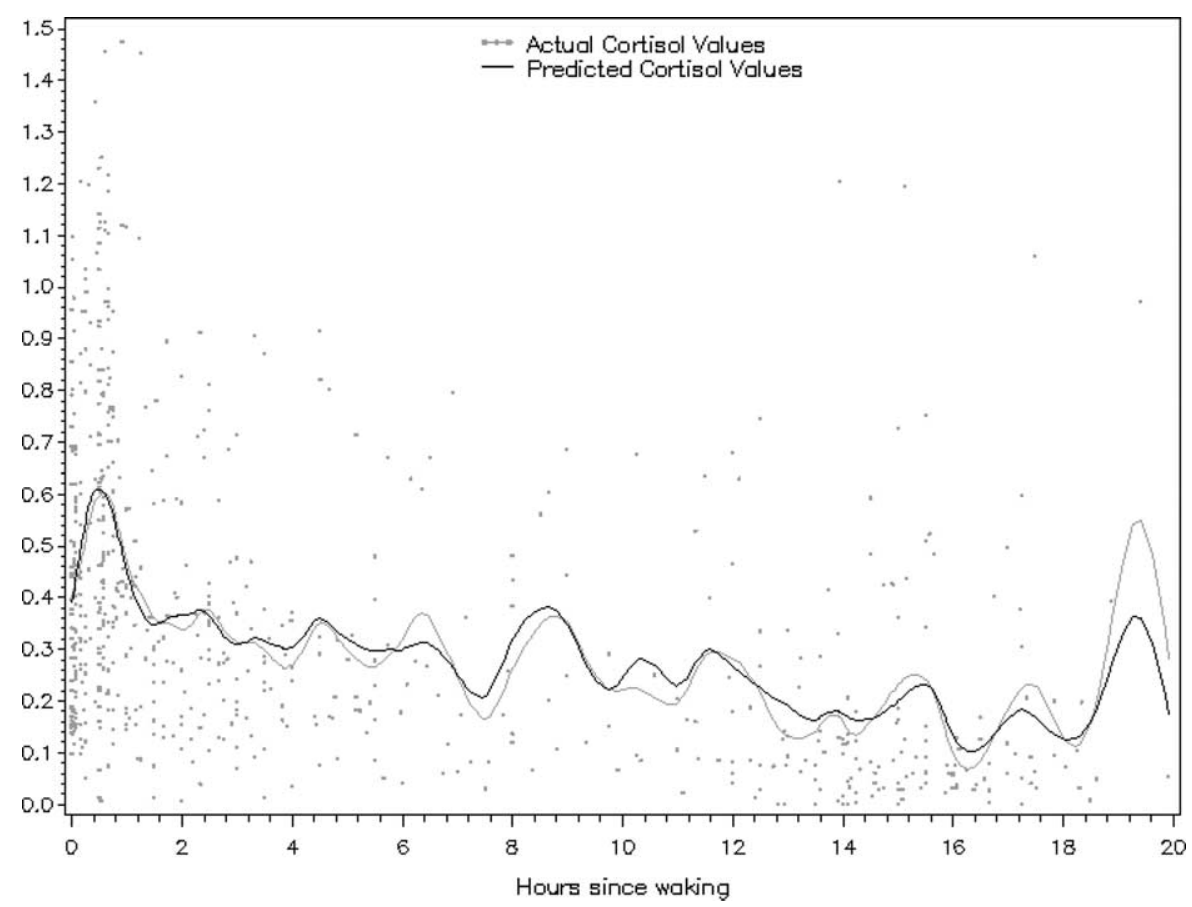

Figure 2 Actual cortisol mean profile versus predicted cortisol mean profile from random effects model with splines. 


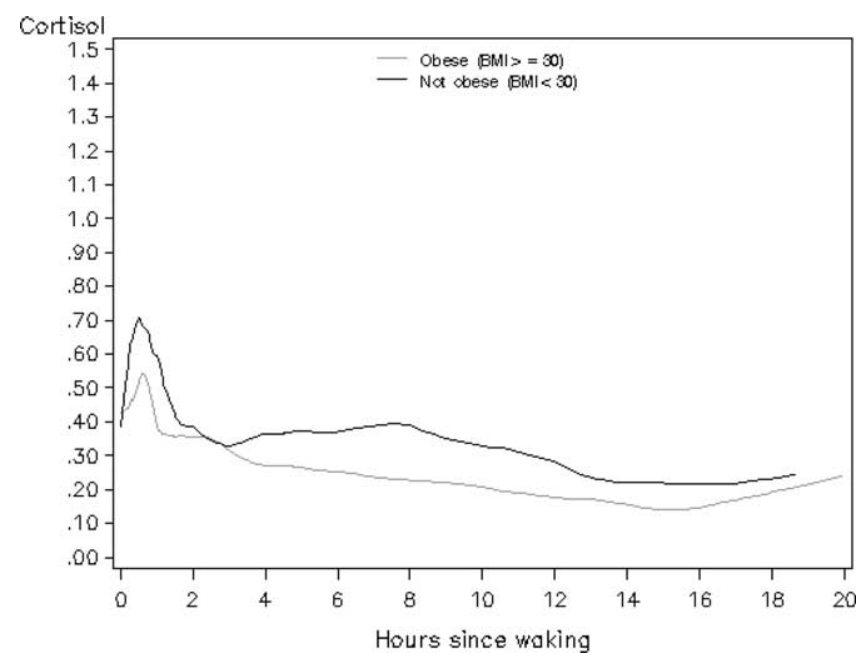

Figure 3 Loess-based diurnal profiles of cortisol in obese and non-obese women.

statistically significant, we fitted a random-effects model that allowed each of the spline slope parameters and the intercept to vary as a function of obesity status. This was achieved by adding a main effect of obesity status, and allowing interaction of the obesity variable with each of the spline parameters.

The estimated parameters by obesity status are presented in Table 2. There is little difference between obese and non-obese women in awakening cortisol level $(0.368$ vs. 0.395$)$, modeled by the intercept in this model. Obese women, relative to non-obese women, have both a shallower slope ( 0.364 vs. $0.638, p=0.0545$ ) for the morning rise, and a slower rate of decline following the morning peak $(-0.268$ vs. -0.382$)$, although this difference is not statistically significant. Over the rest of the day, there are no significant slope differences between obese and non-obese women $(-0.014$ vs. $-0.016)$. However, because of the slower rise and decline in cortisol levels during the awakening surge, the level of cortisol for obese women remain relatively lower than the level for non-obese women, during this time of the day. Thus, the data suggest that obesity may be associated with lowered cortisol levels both during the morning surge, and over the rest of the day. Random effect parameters in this model (not shown) remain largely unchanged from the two previous specifications. Other models we tested, but do not present, do not point to any single characteristic or set of characteristics of obese women that differentiate their parameter variance or parameter mean from those of non-obese women.

\section{Discussion}

This exercise in modeling cortisol data collected over time demonstrates how data collection protocols and modeling techniques can be matched to meet the objectives of an analysis. A key objective for this set of analyses was to demonstrate that such an approach is feasible even under 'real-life' sampling conditions, characterized by poor compliance with both timing protocols and the required number of observations. The inflection points and parameters described here are specific to this sample of poor women aged 19-54, and may not generalize to other population samples with other characteristics. Given the range of time values spanned by our data, the profiles modeled here

Table 2 Implied parameter estimates from random effects model adjusting for obesity effects on intercept and slopes of each segment of the diurnal profile.

\begin{tabular}{lllll}
\hline Parameter & Obesity status & Difference in & $\begin{array}{l}p \text {-Value for } \\
\text { difference }\end{array}$ \\
\cline { 2 - 4 } & Obese women & Non-obese women & $-0.027(0.053)$ & 0.6153 \\
\hline Intercept & $0.368(0.032)$ & $0.395(0.042)$ & $-0.275(0.141)$ & 0.0545 \\
Slope of Phase 1 & $0.364(0.087)$ & $0.638(0.111)$ & $0.115(0.083)$ & 0.1679 \\
Slope of Phase 2 & $-0.268(0.053)$ & $-0.382(0.064)$ & $0.002(0.003)$ & 0.6488 \\
Slope of Phase 3 & $-0.014(0.003)$ & $-0.016(0.003)$ & & \\
Centered age & $-0.050(0.014)$ & $-0.050(0.014)$ & & \\
\hline
\end{tabular}


describe only the waking rhythm of cortisol. The value of these approaches arises from the fact that they can be extended to very different populations and sampling schedules. Depending on data density at different points of the day, the analyses could be extended to incorporate measures such as the mean cortisol value obtained $12 \mathrm{~h}$ after awakening, the slope of decline between various inflection points, area-under-the-curve, or other measure.

Our data collection protocol reflected our modeling objectives. Because non-linear change was expected in the period immediately following awakening, the protocol required two of the four data points to be collected in that period. More spacing was allowed for measurements during the latter part of the day, as less change was expected during that period. The positioning of one or two data point close to bedtime was intended to capture the decline to the evening nadir; as it so happens, this temporal separation of the evening data point from the two morning measurements also has the effect of increasing the precision of the estimates. While a greater number of data points per participant, and a greater stress on compliance, would have yielded greater latitude in modeling, the additional data collection burden on this set of participants would possibly have been too onerous. Other research plans might focus on modeling the slope of the decline between the morning and evening cortisol values; yet, others might aim to obtain reliable estimates of area under the curve. Obviously, data collection protocols would vary accordingly. For instance, researchers interested in studying evening cortisol levels would need more evening samples to be collected. If the morning peak is of interest, as in this case, instructions to participants could possibly stress more compliance during periods of high physiological variation, and be less detailed for periods believed to be more stable. A common practice is to require subjects to collect cortisol data at fixed time points or at fixed intervals from some index time, such as time of awakening or bedtime. Our experience strongly suggests that a more relaxed time schedule is advantageous in terms of modeling, since precision is improved with greater variability of the timepredictor. As cortisol studies over large populations become more common, it is important to arrive at a set of feasible data collection practices.

Our data confirm the presence of a morning rise in cortisol followed by a sharp decline, and a subsequent slower pace of decline to an approximately constant value for the evening hours. The nonlinearities in the data suggest that spline-based regression techniques may be the preferred modeling approach if the intent is to capture variation in the morning changes. Two approaches to modeling spline functions are common in the literature. Nonparametric smooth spline functions offer the highest degree of flexibility, but require large numbers of data points per person. The approach was ruled out in this case, with a maximum of 4 data points per subject. Moreover, it is difficult and impractical to make inferences using models based on non-parametric splines. A second approach, equally data intensive, is to use several 'knots' across the entire profile and to model these parametrically, so as to maximize the chances of capturing non-linearities. We believe that our approach, with its combination of graphical approaches to determine knot location and parametric regression with a limited number of knots, provides much of the modeling flexibility of the above methods, while requiring fewer data points per subject. In the interests of parsimony, another decision we made was to restrict the regression parameters to first-order terms. Polynomial spline models, which use successive powers of the linear spline terms (typically cubic splines) allow for greater smoothness of the curve at the inflections. It did not appear from the graphical analysis that such smoothness was important to model in the case of cortisol profiles.

Whatever the data collection protocol and form of regression specification for the mean profile, random-effects methods for repeated data offer a number of advantages for modeling such data. They allow for differing numbers and spacing of observations across women, while taking into account the correlation of observations within subjects. As long as data points are missing at random, these methods provide unbiased parameters. The fact that the mixed model allows us to use time on a continuous scale, rather than in terms of a sequence of trials, takes advantage of variability in actual measurement time, even when such measurement times depart from instructions. Unlike OLS based models, random-effects models allow relaxation of the assumptions of no-autocorrelation and homoscedasticity. Finally, mixed models allow explicit modeling and analysis of between- and withinindividual variation. Depending on the research focus, the between- and within-variance components of the model may themselves be of interest, or they may be viewed as nuisance parameters.

From our obtained modeling results, it is clear that between-subject variability in cortisol variation is very large, accounting for $62 \% \quad((0.071-$ $0.027) / 0.071$ ) of the total variance, compared to within-subject variance, which accounts for $38 \%$ $(0.027 / 0.071)$ of the total variance. This large variance in cortisol values between subjects, even in a relatively selected sample of women, may have 
possible clinical relevance as well as implications for future modeling efforts. The results suggest that more information can be obtained with fewer data points per subject and larger numbers of subjects. The apparent drift away from the mean over the course of the day, with greater residual variance of evening cortisol values is likely due to the data collection protocol, which resulted in greater sparseness in data for the evening values. This lower density of data for the evening is also reflected in the larger standard error on the interaction parameter for the slope of fourth segment.

Turning to the results of the obesity model, it is apparent that there are small differences in the cortisol profile by obesity status, particularly in the awakening response. The results suggest that both the morning peak and the immediate decline are less marked for obese women. On the other hand, no significant differences between obese and nonobese women were seen in either the awakening value of cortisol, or in the evening level of cortisol, suggesting that area-under-the-curve analyses would have had little utility in distinguishing between obese and non-obese women. Our finding of normal or slightly lower cortisol levels among obese subjects is consistent with recent literature on this subject (e.g. Bjorntorp and Rosmond, 2000; Jessop et al., 2001), but our analysis helps direct attention to the region where this lowering is most marked. Additionally, the inclusion of obesity as an independent subject-specific predictor of cortisol profiles did nothing to explain the variance of cortisol profiles, although there was a substantial effect on the mean parameter values. It is possible that there are other factors that we did not investigate, which account for changes in variance over the course of the day, or that help to discriminate between women with different cortisol levels and profiles.

Despite the interesting findings in this study with respect to obesity and cortisol pattern, we are primarily offering it as an example of some new and relatively simple approaches to analysing a limited number of salivary cortisol measures collected over the day, and caution against generalizing the substantive results. The sample of women is highly selected, and it is possible that the mean profile of this group of women differs from other samples. This group of welfare recipients making the transition to work has been documented elsewhere to suffer high levels of stress and sleep disruption (Presser and Cox, 1997; Zedlewski, 1999), all of which are likely to influence cortisol rhythms. Both experimental and observational studies have found a marked fragmentation of the cortisol quiescent period in response to alteration of sleep cycles
(Caufriez et al., 2002). Further studies are also required to confirm if the inflection points we observed at 30 and $75 \mathrm{~min}$ are common across various exposures, or whether the location of these knots also shifts dramatically with exposure. The models we have used are robust to small shifts in the location of these knots.

The modeling and data collection exercise detailed above may be of value to other researchers in this relatively new field, where there is considerable need for exploring and developing analytic methods, especially at a time when cortisol studies are evolving from small resource-intensive, clinical studies to large-scale population studies.

\section{Acknowledgements}

Data collection and analysis were supported by an award from the National Institute for Child Health and Development (HD P50 HD38986) and by funds from the Michigan Initiative on Inequalities in Health to George A. Kaplan. Major funding of the WES study was provided by the Charles Stewart Mott Foundation and the Joyce Foundation. Elizabeth A. Young is funded by an award from the National Institute of Mental Health (MH01931). We thank the participants in the WES who so generously participated in the study.

\section{Appendix. Abbreviations}

$\begin{array}{ll}\text { HPA } & \text { hypothalamic pituitary adrenal } \\ \text { WES } & \text { Women's Employment Study } \\ \text { WES-HS } & \text { Women's Employment Study-Health } \\ & \text { Supplement } \\ \text { OLS } & \text { ordinary least squares } \\ \text { REML } & \text { restricted maximum likelihood } \\ \text { MLE } & \text { maximum likelihood estimators } \\ \text { BMI } & \text { body mass index } \\ \text { AIC } & \text { Akaike information criterion }\end{array}$

\section{References}

Bjorntorp, P., Rosmond, R., 2000. Obesity and cortisol. Nutrition 16 (10), 924-936.

Caufriez, A., Moreno-Reyes, R., Leproult, R., Vertongen, F., Van Cauter, E., Copinschi, G., 2002. Immediate effects of an 8-h advance shift of the rest-activity cycle on 24-h profiles of cortisol. American Journal of Physiology-Endocrinology \& Metabolism 282 (5), E1147-E1153. 
Danziger, S.K., Seefeldt, K.S., 2002. Barriers to Employment and the 'hard to serve': implications for services, sanctions and time limits. Focus 22 (1), 76-81.

Erosheva, E.A., Greenhouse, J.B., Kroboth, P.D., 2002. Characterizing the diurnal rhythm of DHEA. The American Statistician 56 (4), 273-283.

Hucklebridge, F., Clow, A., Rahman, H., Evans, P., 2000. The cortisol response to normal and nocturnal awakening. Journal of Psychophysiology 14 (1).

Jessop, D.S., Dallman, M.F., Fleming, D., Lightman, S.L., 2001. Resistance to glucocorticoid feedback in obesity. Journal of Clinical Endocrinology \& Metabolism 86 (9), 4109-4114.

King, J.A., Rosal, M.C., Ma, Y., Reed, G., Kelly, T., Stanek, E.J., Ockene, I.A., 2000. Sequence and seasonal effects of salivary cortisol. Behavioral Medicine 2000;.

Kirschbaum, C., Hellhammer, D.H., 1994. Salivary cortisol in psychoneuroendocrine research: recent developments and applications. Psychoneuroendocrinology 19, 313-333.

Kudielka, B.M., Broderick, J.E., Kirschbaum, C., 2003. Compliance with saliva sampling protocols: electronic monitoring reveals invalid cortisol daytime profiles in noncompliant subjects. Psychosomatic Medicine 65 (2), 313-319.

Presser, H.B., Cox, A.G., 1997. The work schedule of loweducated American women and welfare reform. Monthly Labor Review 120, 25-34.

Pruessner, J.C., Hellhammer, D.H., Kirschbaum, C., 1999. Burnout, perceived stress, and cortisol responses to awakening. Psychosomatic Medicine 61 (2), 197-204.

Pruessner, J.C., Kirschbaum, C., Meinlschmid, G., Hellhammer, D.H., 2003. Two formulas for computation of the area under the curve represent measures of total hormone concentration versus time-dependent change. Psychoneuroendocrinology 28 (7), 916-931.

Reynolds, R.M., Syddall, H.E., Walker, B.R., Wood, P.J., Phillips, D.I., 2003. Predicting cardiovascular risk factors from plasma cortisol measured during oral glucose tolerance tests. Metabolism 52 (5), 524-527.

Schmidt-Reinwald, A., Pruessner, J.C., Hellhammer, D.H., Federenko, I., Rohleder, N., Schurmeyer, T.H., Kirschbaum, C., 1999. The cortisol response to awakening in relation to different challenge tests and a 12-hour cortisol rhythm. Life Sciences 64 (18), 1653-1660.

Sephton, S.E., Sapolsky, R.M., Kraemer, H.C., Spiegel, D., 2000. Diurnal cortisol rhythm as a predictor of breast cancer survival. Journal of the National Cancer Institute 92, 994-1000.

Steptoe, A., Cropley, M., Griffith, J., Kirschbaum, C., 2000. Job strain and anger expression predict early morning elevations in salivary cortisol. Psychosomatic Medicine 62 (2), 286-292.

Wang, Y., Brown, M.M., 1996. A flexible model for human circadian rhythms. Biometrics 52, 588-596.

Yehuda, R., Halligan, S.L., Yang, R.K., Guo, L.S., Makotkine, I., Singh, B., Pickholtz, D., 2003. Relationship between 24-hour urinary-free cortisol excretion and salivary cortisol levels sampled from awakening to bedtime in healthy subjects. Life Sciences 673 (3), 349-358.

Young, E.A., Carlson, N.E., Brown, M.B., 2001. Twenty-four-hour ACTH and cortisol pulsatility in depressed women. Neuropsychopharmacology 25 (2), 267-276.

Zedlewski, S.R., 1999. Work activities and obstacles to work among TANF recipients. The Urban Institute National Survey of American Families; Series B (B-2).

Available online at www.sciencedirect.com

SCIENCE 0 DiRECT 